\title{
Periodismo de confirmación vs. Desinformación: Verificado18 y las elecciones mexicanas de 2018
}

\author{
Fact-checking vs. Disinformation: Verificado18 and 2018 Mexico's \\ elections
}

Dr. Amaya Noain Sánchez, Universidad Complutense, Madrid (España) amaya.noain@unir.net|http://orcid.org/0000-0001-6910-6346

\begin{abstract}
Resumen
La proliferación de noticias falsas en redes sociales constituye un desafío no sólo para el mundo del periodismo, sino también para nuestras democracias. Este hecho es especialmente controvertido si tenemos en cuenta que estas herramientas se han convertido en una popular fuente informativa, toda vez que los medios de comunicación tradicionales han perdido su supremacía a la hora de seleccionar contenidos noticiables. Como consecuencia, los ciudadanos están expuestos a informaciones sesgadas que promueven la desinformación. Particularmente ilustrativo, es el papel desempeñado por Facebook y Twitter en la divulgación de afirmaciones falsas de políticos durante varias de las campañas electorales de los últimos años. Empero, fue el flujo de desinformación surgido durante las elecciones presidenciales estadounidenses de 2016 el detonante que alimentó el interés en chequear todos aquellos contenidos distribuidos a través de Internet. En este contexto, el objetivo de la investigación es analizar el trabajo desarrollado por las iniciativas de periodismo de comprobación de datos, centrándonos en el caso de Verificado18: un proyecto colaborativo iniciado en México en 2018, de cara a las elecciones más multitudinarias del país y destinado a identificar promesas falsas y contenidos manipulados. Para ello, realizamos un abordaje tanto cuantitativo como cualitativo de los datos extraídos de la Web verificado.mx. Al hacerlo, arrojaremos luz sobre aspectos como: la metodología utilizada, las temáticas cubiertas, los actores involucrados y las herramientas interactivas implementadas. Los hallazgos sugieren que, a pesar de su potencial educativo, es pertinente mejorar aspectos como la transparencia.
\end{abstract}

Forma de citar:

Noain Sánchez, A. (2019). Periodismo de confirmación vs. Desinformación: Verificado18 y las elecciones mexicanas de 2018. Ámbitos. Revista Internacional de Comunicación 43(1), 95-114. doi: 10.12795/Ambitos.2019.i43.05 


\begin{abstract}
The increase in the number of fake news on Social Network Sites (SNS) form a serious threat not only to journalism, but also to our democracies. This is not a minor issue, given that SNS has become into a popular source of information, whilst, at the same time, traditional media are declining in their gatekeeping role. As a result, citizens are exposed to biased news that lead to disinformation. An illustrative example is the role played by Facebook and Twitter in spreading false or outrageous statements by politicians during some of the latest political campaigns. Nonetheless, it was the flow of disinformation around the 2016 US presidential election that spurred intense interest in fact-checking items of news spread online. In this context, the aim of this paper is to analyse the work undertaken by fact-checking outlets, by focusing on Verificado18: a collaborative project launched in 2018 ahead of Mexico's largest election, to identify untrue statements from politicians and check content authenticity. To fulfil this purpose, we provide this analysis on the basis of a mix of qualitative and quantitative data from verificado.mx. In doing so, we throw light on the methodology used to debunk disinformation, the topics most commonly covered, the actors involved, and the interactive tools implemented. Our findings suggest that, in spite of its educative potential, more efforts needs to be put as far as transparency is concerned.
\end{abstract}

Palabras clave: Noticias falsas; Desinformación; Periodismo de comprobación; Elecciones mexicanas; Verificado18.

Key Words: Fake News; Disinformation; Fact-Checking; Mexico's Elections; Verificado18.

\title{
1. INTRODUCCIÓN
}

La arena pública proporcionada por las redes sociales está teniendo un papel protagónico en muchas de las cuestiones que afectan al correcto funcionamiento de nuestras democracias. En concreto Facebook, la red social creada por Zuckerberg en 2004, se ha consolidado como "medio de representación y espacio de circulación de significado en la sociedad" (Mathieu, 2015:14) y a su vez, Twitter, el servicio de mircroblogging alumbrado en 2006 por Jack Dorsey se ha establecido como herramienta mediadora que da acceso al debate político y es capaz de provocar acciones reactivas (Jungherr, 2015). Es por ello que la divulgación de contenidos falsos y la subsiguiente creación de filtros en estos nuevos espacios de representación de la esfera pública, espacio de creación de la opinión, impone altos costes en la sociedad: generan una alteración de la percepción para la cual el individuo se halla desprovisto de herramientas o referentes de contextualización, dificultando que pueda inferir las informaciones contrastadas de las que no lo están. El alto grado de retroalimentación de esta dinámica, propiciada por la rápida propagación de 
contenidos maniqueos, constriñe nuestras elecciones a un entramado de productos prefabricados que-limitan nuestro acceso a la información plural.

Una de las muestras más llamativas de esta problemática la encontramos en la difusión de información política y, más concretamente, en aquellos contenidos propalados durante procesos electorales y referéndum recientes como, por ejemplo, el Brexit, las elecciones estadounidenses o la campaña por la independencia catalana. Este reto no conforma una tendencia pasajera: en 2022 el público occidental consumirá más noticias falsas que verdaderas y no habrá suficiente capacidad ni material, ni tecnológica, para eliminarlas (Panetta, 2017). En suma, no sorprende que la desinformación y la proliferación de estas noticias apócrifas, malintencionadas o imprecisas constituyan un desafío para las democracias, convirtiéndose en foco de interés para académicos. Es en este escenario donde los proyectos de periodismo de confirmación de datos, en sus diversas versiones y con sus múltiples orígenes, emergen como una alternativa viable para luchar contra la adulteración de la información.

Para aproximarnos a su idiosincrasia, el presente texto analiza la metodología pragmática de la plataforma mexicana Verificado18, nacida al albor de las elecciones presidenciales celebradas en el país en 2018 y destinada a examinar informaciones y declaraciones dudosas referentes a los comicios. En el estudio se establece una distinción entre las dos funcionalidades básicas observadas en el periodismo de datos, a saber: la contrastación de informaciones y su vertiente contextualizadora y explicativa del escenario en el que aparece la información. Enlazada a esta doble función, asumimos la siguiente hipótesis:

"Existe una relación directamente proporcional entre la capacidad para desbancar las noticias falsas y a) la transparencia en los procesos de comprobación, esto es, a su capacidad para rendir cuentas, b) la difusión óptima de los resultados".

Para dar respuesta a dicha afirmación, partimos de una aproximación descriptiva de los procesos de verificación, para, posteriormente, aplicar una metodología mixta, tanto cuantitativa como cualitativa, en la que examinamos la pertinencia de esta iniciativa en el citado escenario informativo, las temáticas cubiertas, los contenidos generados y la divulgación de dichos resultados, incluyendo las herramientas interactivas utilizadas para conectar con el ciudadano.

En última instancia, el objetivo es evaluar la efectividad de este proyecto para ofrecer referentes de interpretación del escenario electoral, ahondando en su labor en favor de la pluralidad informativa, la diversidad de ideas y la veracidad de los datos, puntos clave del debate público. 


\section{REVISIÓN TEÓRICA Y PLANTEAMIENTO DEL PROBLEMA}

La inserción y divulgación de bulos, rumores y medias verdades bajo la apariencia de información no es una práctica nueva, sino que forma parte de la propia historia de la propaganda. En épocas convulsas, muchos gobiernos han hecho uso de esta estrategia para distorsionar la percepción de la realidad y generar confusión en la sociedad. No se trata, por tanto, de sesgos ideológicos enlazados a líneas editoriales como los que podemos encontrar en los medios de comunicación tradicionales, sino de estrategias encaminadas a incidir en los resultados electorales, en las políticas de un determinado país e, incluso, desestabilizar regímenes.

El término "noticias falsas" se popularizó en 2016 durante las elecciones presidenciales estadounidenses, coyuntura en la que el propio presidente de Estados Unidos, entonces candidato, hizo uso cuantioso del vocablo, aunque para criticar a todos aquellos medios que publicaban noticias que no eran de su agrado. Dicha voz refiere a "artículos que son intencionalmente y verificablemente falsos y pueden engañar a los lectores" (Allcott \& Gentzkow, 2017). Junto a este vocablo, expresiones como "posverdad" (Tesich, 1992) sirvieron para expandir una retórica que, al legitimar el fenómeno como novedad, infravaloraba y simplificaba la verdadera naturaleza de la cuestión: la desinformación estructural en la red (Comisión Europea, 2018), una problemática de raíces más profundas y en la que las noticias falsas constituyen meramente la punta de iceberg.

\subsection{El entramado de la desinformación Internet}

Las redes sociales se han convertido en una de las principales fuentes informativas, por delante de los medios tradicionales. Por citar un ejemplo paradigmático, durante las elecciones presidenciales estadounidenses de 2016, estas herramientas constituyeron la primera fuente de información para un $62 \%$ de la población adulta estadounidense. Esta cifra, además, esconde una tendencia al alza, en tanto que plasma un crecimiento respecto a los pasados comicios de 2012, año en que se situaba en el 49\% (Gottfried \& Shearer, 2016). No obstante, y aunque se distribuya bajo el emblema de "noticia", no todo aquello que se divulga a través de estos canales responde a los criterios clásicos de rigurosidad y veracidad de la información.

Desde el punto de vista de los medios de comunicación, la divulgación de contenidos no confiables o incorrectos tiene su origen, primordialmente, en condicionamientos económicos. En primer lugar, numerosos medios, incluso aquellos consagrados, son susceptibles de caer en el error de difundir contenidos no contrastados movidos por la premura que imprime la actualidad y la simultaneidad que caracteriza al periodismo digital (Loreto-Echeverri, Romero-Rodríguez \& Pérez-Rodríguez, 2018:297). En segundo lugar, encontramos la manipulación de contenidos, destinada a producir noticias afines a la línea editorial o que reafirmen un determinado ideario y, 
con ello, de nuevo la sombra de los imperativos económicos. Finalmente, la proliferación del clickbait, una práctica controvertida ligada a las líneas de financiación de los medios digitales y que traspasa las demarcaciones éticas deseables (Restrepo, 2017). Mediante dicha técnica, algunos medios digitales se especializan en el uso de titulares llamativos, sensacionalistas y engañosos, destinados a aumentar el número de visitas que reciben, en un intento por buscar la viralización de sus contenidos antes que su función informativa (Palau-Sampio, 2016).

Empero, si los contenidos adulterados y apócrifos encuentran en los mecanismos de distribución de las redes sociales digitales un terreno propicio para su rápida divulgación también es debido, en parte, a la interacción de agentes que nada tienen que ver con la práctica periodística. En esta dinámica intervienen varios factores: En un extremo de la ecuación, encontramos a los usuarios, quienes tienen libertad para crear contenidos, acceder a las publicaciones que más les interesa y decidir qué comparten con sus contactos. Es cierto que la audiencia juega un role más activo y está más involucrada en el circuito mediático que en los medios clásicos, aunque sólo uno de cada ocho usuarios crea nuevos materiales y lo hace en forma de fotos (14\%) o vídeos (12\%) (Anderson \& Caumont, 2014). Centrándonos en los ítems de corte informativo, los usuarios también discuten (46\%) y comparten (50\%) noticias (Anderson \& Caumont, 2014) y dentro de estas publicaciones, los contenidos falseados son los más ampliamente compartidos (Silverman, 2016). Dado que las publicaciones maniqueas apelan a sentimientos como la indignación o la ira y tienen una función más expresiva que informativa (Gallant, Latzo-Toth \& Pastinelli, 2015) se viralizan rápidamente, provocando adherencia y reforzando creencias preexistentes (Shin, Jian, Driscoll \& Bar, 2018; Tufekci, 2015). En el polo opuesto, advertimos que estos materiales provienen de un espectro de fuentes muy limitado que pueden catalogarse en dos tipos: a) emisores que se mueven por motivaciones económicas, es decir, orientados a conseguir beneficios monetarios pero sin filiación ideológica de ningún tipo y, en menor medida, b) aquellos con motivaciones políticas (Allcott \& Gentzkow, 2017). Estos últimos, pueden estar organizados, esto es, grupos de personas que se dedican exclusivamente a crear y difundir contenidos sesgados, o bien provenir de usuarios individuales que se adhieren a una determinada corriente. En el primero de los casos, encontramos proveedores de contenidos (Sydell, 2016) y usuarios independientes, como, por ejemplo, el grupo de estudiantes de Macedonia que, gracias a sus publicaciones falseadas en apoyo a Trump, consiguió obtener publicidad para sus más de cien sitios web (Subramanian, 2017). En el segundo, tenemos a los propios líderes, asesores y otros grupos de apoyo que usan la red para congregar a sus adeptos en torno a sus ideales, tal y como sucedió en la campaña pro-Brexit (Viner, 2016) o pro-Trump (Townsend, 2016). Todo ello, sin olvidar las empresas que 
realizan campañas encubiertas a favor de un determinado ideario o líder político, hecho ya observado en los citados comicios, en el referéndum del Brexit o en el proceso catalán (ICA, 2017; Danzig, 2017). Estas taxonomías, no obstante, no conforman compartimentos estancos sino que, a menudo, se entremezclan ambas motivaciones.

Ante esta situación, las iniciativas de periodismo de confirmación vienen a retomar una máxima de la profesión que consiste en pasar por el tamiz de la verificación la autenticidad de la información proveniente de los poderes establecidos, flanco al que ahora se suman la amalgama de contenidos dudosos que campan a sus anchas por las redes sociales.

\subsection{El periodismo de confirmación}

El "periodismo de confirmación", "chequeo", "comprobación de datos" o "Fact-ckecking", hace su aparición en Estados Unidos a través de FactCheck.org, proyecto creado en 2003, dentro del Annenberg Public Policy Center de la University of Pennsylvania. Desde entonces, han sido muchas las plataformas destinadas a promover un "estilo periodístico capaz de auscultar la veracidad de las promesas políticas" (Gentzkow, Jesse \& Daniel 2016:1). Si bien es cierto que la confrontación de fuentes y verificación de datos es una labor indisoluble en las redacciones de los medios de comunicación, pues constituye la base de la deontología periodística, los proyectos de periodismo de comprobación no surgen para sustituir dichas secciones, sino que completan la cadena periodística a posteriori, actuando como centro de apoyo capaz de concentrar las demandas de verificación del gran público e investigar aquellos contenidos inciertos que, no necesariamente, provienen de profesionales de la información.

Así pues, el valor concreto de esta reinvención del periodismo tradicional consiste en su capacidad para dar respuesta al doble desafío que presenta la desinformación, esbozando un enfoque integrador que contempla, de un lado, una dimensión de acceso a información contrastada y veraz y, de otro, la expansión de la alfabetización mediática tan necesaria para desarrollar un pensamiento crítico. Tras esta primera iniciativa, emergen otras plataformas de chequeo vinculadas a medios en Estados Unidos: en 2007, ve la luz PolitiFact, vinculado a St. Petersburg Times (actualmente Tampa Bay Times, parte de Poynter Institute) y The Washington Post's Fact Checker. Y en Europa, diarios como Libération, Der Spiegel y cadenas de televisión como Channel 4 o La Sexta hicieron lo propio.

Estos proyectos se generan de muy diversas formas, por lo que la heterogeneidad que se desprende de semejante mezcolanza dificulta su clasificación. Sin embargo, a pesar de desarrollar su labor de maneras distintas y presentar estructuras organizativas variadas, esta miscelánea tiene en común la necesidad de promover el discurso democrático y un gobierno capaz de rendir 
cuentas (Graves, 2018: 14). En un intento de establecer una suerte de clasificación, Graves y Cherubini definen una taxonomía básica en la que diferencian entre: a) proyectos que surgen como una sección autónoma dentro de un medio de comunicación y que, si bien cuenta con más recursos técnicos y económicos, pueden verse lastrados por la lógica empresarial (Graves \& Cherubini, 2016: 8-10); b) un reporterismo alternativo, sin ánimo de lucro, que se financia a través de fundaciones y que, a pesar de coaligarse con medios ya establecidos para su divulgación, mantiene su independencia, lo que le permite un margen más amplio de actuación (2016:1011). Mientras que el primero de los esquemas es característico en Europa occidental y Estados Unidos, en Latinoamérica y Europa del Este prolifera el segundo.

\subsection{Pluralismo informativo en Latinoamérica y génesis del movimiento Verificado18}

La aparición del periodismo de comprobación en Latinoamérica representa no sólo la respuesta a una problemática contemporánea de calado internacional, esto es, la desinformación endémica en redes sociales, sino la solución ante una estructura de medios tradicionalmente marcada por el oligopolio y el inmovilismo. En las pasadas décadas, la llegada de Internet abrió nuevos horizontes en una región donde grupos mediáticos controlaban la información, cimentando, la emergencia de proyectos de periodismo de chequeo, una clara tendencia al cambio. Estas plataformas han seguido los pasos de la argentina Chequeado, modelo iniciador en el continente en el año 2010. Desde entonces, han prosperado, entre otras: Detector de Mentiras y ColombiaCheck en Colombia; Truco, Agência Lupa, Aos Fatos y Agência Pública en Brasil; El Sabueso y ChecaDatos. $m x$ en México y Con pruebas en Guatemala. El fenómeno se está estableciendo en la región donde, hasta Febrero de 2018, se contabilizaban 15 de las 149 iniciativas activas en todo el mundo, incremento a tener en consideración dado que 2014 sólo había tres (Stencel \& Griffin, 2018).

La aparición de la mexicana Verificado18 va enlazada a los comicios de mayor magnitud hasta la fecha, en los que, además de disputarse la Presidencia de la República, también estaban en juego el Congreso y nueve gobiernos estatales. En total, más de 3400 cargos de elección popular en manos de los votos de casi 88 millones de personas (Najar, 2018). Este servicio de periodismo colaborativo nace el 12 de marzo de 2018, a propuesta de Animal Político ${ }^{1}$, Newsweek en Español, Pop Up Newsroom y AJ+ Español. En total, aglutina a más de sesenta medios de comunicación de todo el país, universidades y fundaciones. Corresponde, por tanto, al segundo modelo dentro de la clasificación descrita por Graves y Cherubini (2016: 10-11) ya que no nace dentro de la redacción de un medio concreto. 
El nombre del proyecto no fue escogido al azar, sino que proviene de un movimiento surgido tras el terremoto que sacudió México el 19 de Septiembre de 2017. La mañana siguiente al sismo, a iniciativa ciudadana, surgió el hashtag "\#Verificado19S", creado para proveer a los rescatistas y hacer frente a la divulgación de informaciones erróneas que sólo servían para confundir y atemorizar a la población. Al igual que entonces, la información falaz persigue "un sentido parecido al que se enfrentó en el temblor: confunde, paraliza, asusta. Busca, en ese sentido, socavar la libertad del ciudadano para decidir por quién votar" (Khatib, D. y Larrea Maccise, 2018)

Entre sus peculiaridades, llama poderosamente la atención el hecho de que esta iniciativa reciba el apoyo de Facebook (Facebook Journalism Project) Twitter y Google (Google News Initiative) entidades que, junto con organizaciones de la sociedad civil, como Mexicanos Contra la Corrupción y la Impunidad, y fundaciones como Open Society y Oxfam, financian al equipo de periodistas que trabajarán en la redacción (Verificado, 2018: Web). Asimismo, a pesar de no estar adscrita a un medio concreto, posee una estructura permanente en la que destaca la redacción multimedia, lo que le permite elaborar materiales audiovisuales destinados a la contextualización del panorama político, así como a impulsar la interactividad con los usuarios. Finalmente, otro apunte novedoso es la importancia que se da a los contenidos divulgados por medio de WhatsApp, sustrato donde la difusión de noticias falsas provoca efectos más profundos, dado que se trata de contenidos altamente individualizados y que se introducen dentro del espectro privado del individuo, por lo que la divulgación de su corrección es más complicada (Hazard, 2018). Esta particularidad sitúa el modelo Verificado18 como un referente a seguir por otros países como, por ejemplo, la India, donde los 250 millones de usuarios de WhatsApp reciben infinidad de materiales manipulados a través de este sistema (Goel, 2018).

\subsection{Metodología}

Partiendo de una aproximación descriptiva, se aplica una metodología mixta que combina técnicas cuantitativas y cualitativas. Como unidades de análisis, delimitamos las entradas publicadas en el sitio verificado.mx, compiladas con el propósito de identificar qué publicaciones corresponden a: 1) confirmación de datos y comprobación del discurso; 2) creación de materiales explicativos. Esta distinción establece una primera correlación entre las dos funcionalidades básicas observadas en el periodismo de datos. Asimismo, se registran todos los post de sus redes sociales, así como las repuestas generadas por los usuarios a través de sus distintas etiquetas, como apoyo a la delimitación de ejes temáticos y evaluación de la capacidad de interacción y divulgación. El análisis comprende los contenidos producidos por la plataforma desde su lanzamiento, el día 12 de marzo, hasta el 9 de julio, momento en que cesa su actividad. El corpus 
de estudio lo conforman un total de 356 ítems, cuyo análisis nos permite evaluar: a) metodología y proceso de verificación; b) principales ejes temáticos; c) resultado de las comprobaciones y correspondencia con los actores implicados; d) presentación y divulgación de los resultados, e) interactividad y elementos multimedia.

\section{RESULTADOS}

\subsection{Metodología y proceso de verificación}

La plataforma Verificado2018 persigue una doble finalidad: "hacer cara a la difusión de contenidos falseados y poner el contrapunto a todas aquellas promesas irrealizables, denuncias sin fundamento y críticas basadas en datos equivocados" (Khatib \& Larrea Maccise, 2018: Web). Dicha propuesta se materializa en dos líneas de acción que, a su vez, estructuran su página Web en dos grandes bloques: a) el apartado "Noticias falsas", destinado a la comprobación de datos y confrontación de fuentes; y b) "Verificaciones", centrado en la vigilancia del poder a través de la auscultación de las promesas electorales. Existe, igualmente, una sección más pequeña, "Multimedia", destinada a tutoriales, cobertura de debates y recursos de cariz explicativo y educativo. En total, los 352 contenidos publicados en el portal se distribuyen entre: 153 publicaciones correspondientes a "Noticias falsas"; 163 "Verificaciones"; diez entradas que conjugan ambas etiquetas y 26 post correspondientes al apartado "Multimedia".

Poniendo el foco en los procesos de comprobación llevados a cabo, según Verificado18 se aplica una metodología distinta en función de la sección a la que se adscriben:

A) La sección "Noticias falsas" parte de una selección de ítems informativos, de muy diverso calado (artículos, fotos, vídeos, carteles electorales, gifts...) y contenido dudoso, que se investigan una vez hayan alcanzado más de mil interacciones, por lo que también contempla bots o cuentas automatizadas. Igualmente e independientemente del número de interacciones, se examinan rumores o afirmaciones que, debido a su viralidad en redes sociales y medios tradicionales, requieran ser probados. Una vez seleccionados, la comprobación consta de seis pasos: 1) revisión de la fuente de publicación; 2) contrastación de datos y búsqueda de originales en el caso de formatos audiovisuales; 3) ratificación con los actores involucrados; 4) consulta a otras fuentes como, por ejemplo, testigos o asistentes a los eventos; 5 ) escrutinio de datos estadísticos y académicos relacionados con la temática; 6 ) capturas de pantalla de las noticias falsas (aunque nunca se publican enlaces a los sitios que las difunden). Tras dicho proceso y haciendo uso de la categorización ya utilizada por Chequeado, se dictamina a qué categoría se circunscribe cada ítem, valorándolo como: “Falso", "Verdadero", “Engañoso" o "No se puede probar”. 
B) En lo que atañe a la verificación del discurso, esta se basa en el sistema de El Sabueso², metodología consta de siete pasos y las siguientes taxonomías: "Verdadero", "Verdad a medias", "Discutible", "No se puede probar", "Engañoso", "Casi falso", "Falso" o "Ridículo".

La frecuencia de las comprobaciones en dichos bloques es diaria, salvo en el caso de los tres debates presidenciales, en los que el discurso de los candidatos se analiza en tiempo real durante la celebración de los mismos.

Respecto al tratamiento metodológico de estos dos primeros apartados, cabe señalar una primera aclaración: si bien se arguye la aplicación de procedimientos de comprobación diferenciados en función de la naturaleza del contenido, esto es, dependiendo de si nos referimos a ítems informativos o a declaraciones, en la práctica esta clasificación no se respeta. Por consiguiente, al igual que la aplicación de ambas metodologías es arbitraria, también lo son los dictámenes que categorizar cada contenido evaluado por el quipo de la plataforma.

Contabilizando las entradas destinadas al chequeo de contenidos, 202 en total, encontramos: 146 informaciones con la etiqueta "Falso" (72,27\%), 37 catalogadas como "Verdadero" y/o "\#EstoSíPasó" (18,31\%), 6 como "Engañoso" (2,97\%) y 3 que "no se pueden probar" $(1,48 \%)$. Así pues, los resultados señalan que la mayoría de los enunciados pueden catalogarse como "verdaderos" o "falsos".

\subsection{Principales ejes temáticos}

Los contenidos que vertebran parte de la actualidad informativa, como son: las declaraciones e informaciones atribuidas a representantes del ámbito político $(32,2 \%)$, las acciones supuestamente desarrolladas por los partidos $(14,37 \%)$, la publicación de encuestas sospechosas $(7,83 \%)$ y las irregularidades en el proceso electoral $(5,88 \%)$ son aquellos más abundantemente sometidos a examen.

Más concretamente, en el caso de los elegibles, un 19,6\% de las informaciones refieren a la revelación de información controvertida sobre dichas personas, tanto dentro como fuera del foco mediático, mientras que un $16,99 \%$ provienen de propuestas o declaraciones atribuidas a los mismos. Dichos contenidos se distribuyen en forma de: carteles electorales trucados, entrevistas que nunca se realizaron, memes, vídeos manipulados y montajes fotográficos que, mediante el uso de materiales de otras épocas insertados en el contexto actual, recrean encuentros con otros políticos o caracterizan a sus protagonistas desprovistos de autoridad e, incluso, dignidad (borrachos, por ejemplo). Y, entre estos, destacan aquellas revelaciones sobre supuestos delitos cometidos por los candidatos, así como sus vínculos con entornos criminales, polémica que 
protagonizan el $7,18 \%$ de los artículos. En el lado contrario, la utilización que algunos candidatos hacen de encuestas, datos y otros materiales fraudulentos en beneficio propio alcanza el 6,53\%. Por su parte, el entorno de los candidatos también es objeto de conjeturas con 2,61\%.

Otras informaciones susceptibles de comprobación son aquellas referidas al posicionamiento de personajes mediáticos y de la sociedad civil a favor de un candidato. Este grupo heterogéneo comprende: figuras del mundo del espectáculo (5,88\%), grupos empresariales/empresarios $(3,26 \%)$, medios extranjeros $(4,57 \%)$, autoridades del mundo académico $(1,3 \%)$, miembros de la sociedad civil $(1,3 \%)$, líderes extranjeros $(0,65 \%)$, ejército $(0,65 \%)$ y líderes religiosos como el Papa $(0,65 \%)$.

En lo que atañe al uso de los medios sociales, se divulga la denuncia de plataformas destinadas a la propagación de artículos falseados, así como de perfiles de Facebook y cuentas de Twitter que no pertenecen a los candidatos. Finalmente, Ilama la atención la aparición de temáticas ya vistas en otros procesos electorales como son la injerencia rusa $(1,96 \%)$ y el robo de datos $(0,65 \%)$.

Tabla 1. Ejes temáticos

\begin{tabular}{|l|r|r|r|}
\hline Principales temáticas sometidas a comprobación & Total & Verdaderas & Falsas \\
\hline $\begin{array}{l}\text { Declaraciones e informaciones atribuidas a candidatos } \\
\text { / políticos }\end{array}$ & $32,20 \%$ & $20,40 \%$ & $79,59 \%$ \\
\hline $\begin{array}{l}\text { Posicionamiento a favor de un candidato/ formación } \\
\text { política }\end{array}$ & $18,26 \%$ & $28,57 \%$ & $71,42 \%$ \\
\hline $\begin{array}{l}\text { Acciones/ propuestas atribuidas a una formación } \\
\text { política }\end{array}$ & $14,37 \%$ & $31,82 \%$ & $68,18 \%$ \\
\hline $\begin{array}{l}\text { Irregularidades en el proceso electoral / falseamiento } \\
\text { de encuestas }\end{array}$ & $13,71 \%$ & $33,34 \%$ & $66.66 \%$ \\
\hline $\begin{array}{l}\text { Uso de información fraudulenta por parte de un } \\
\text { candidato en su beneficio }\end{array}$ & $6,53 \%$ & $20 \%$ & $80 \%$ \\
\hline $\begin{array}{l}\text { Declaraciones atribuidas al familiar de un candidato o } \\
\text { líder político }\end{array}$ & $5,88 \%$ & $11,12 \%$ & $88,88 \%$ \\
\hline Otros a los & $1,96 \%$ & 0 & $100 \%$ \\
\hline Disturbios relacionados con la campaña & $1,96 \%$ & 0 & $100 \%$ \\
\hline $\begin{array}{l}\text { Falsos perfiles de redes sociales atribuidos } \\
\text { candidatos }\end{array}$ & $1,30 \%$ & 0 & $100 \%$ \\
\hline Injerencias extranjeras/ gobierno ruso & $0,65 \%$ & 0 & $100 \%$ \\
\hline $\begin{array}{l}\text { Desmentido de informaciones falsas atribuidas a } \\
\text { Verificado18 }\end{array}$ & 0 & $100 \%$ \\
\hline Ataques informáticos/ robo de datos & $3,26 \%$ & $60 \%$ \\
\hline
\end{tabular}

Fuente: elaboración propia con datos de verificado.mx 


\subsection{Actores y resultado de las comprobaciones}

Más de la mitad de los contenidos dudosos corresponden a responsables del gobierno, candidatos elegibles y diputados $(50,32 \%)$ y, entre estos, los candidatos a los distintos cargos conforman el $45,75 \%$ de los exámenes realizados. El segundo puesto del ranking aparece copado, de manera más genérica, por los grupos políticos (14,37\%). Y, dado que las irregulares electorales contituyen una temática candente, el Instituto Nacional Electoral (INE) y otros actores relacionados con el proceso electoral alcanzan el 6,53\% del total. En menor escala encontramos gobernadores, alcaldes y otros responsables de la administración regional y local $(8,49 \%)$. También, medios de comunicación y otras plataformas de distribución de la información, entre las que destaca el papel jugado por medios de relevancia internacional, como The New York Times (3,92\%) y por páginas ya existentes o bien nacidas al albor de las elecciones y destinadas a confeccionar y distribuir información no confiable $(1,96 \%)$. Como ya se ha visto en procesos anteriores, prevalece la importancia de desarrollos como las redes sociales $(5,88 \%)$ y la aparición de las agencias rusas (1,3\%); así como la entrada en escena de figuras del mundo del espectáculo $(4,57 \%)$, grupos empresariales $(3,26 \%)$, líderes extranjeros $(1,3 \%)$, autoridades del mundo académico $(1,3 \%)$, ONG $(1,3 \%)$ y líderes religiosos $(0,65)$ que típicamente son protagonistas subsidiarios en las campañas electorales.

Tabla 2. Actores

\begin{tabular}{|l|r|r|r|}
\hline $\begin{array}{l}\text { Actores de los contenidos sometidos a } \\
\text { verificación }\end{array}$ & \multicolumn{1}{l|}{ Total } & Verdaderas & \multicolumn{1}{l|}{ Falsas $^{3}$} \\
\hline $\begin{array}{l}\text { Representantes del gobierno, candidatos y } \\
\text { diputados }\end{array}$ & $50,32 \%$ & $25,97 \%$ & $68,83 \%$ \\
\hline Partidos políticos & $14,37 \%$ & $31,81 \%$ & $68,18 \%$ \\
\hline INE / actores relacionados con el proceso electoral & $13,72 \%$ & $52,38 \%$ & $47,61 \%$ \\
\hline Otros cargos / administración regional/local & $8,49 \%$ & 0 & $100 \%$ \\
\hline Medios de comunicación / Otras plataformas & $7,84 \%$ & 0 & $100 \%$ \\
\hline Personajes del espectáculo y la cultura & $4,57 \%$ & 0 & $100 \%$ \\
\hline Grupos empresariales / Empresarios & $3,26 \%$ & 0 & $100 \%$ \\
\hline Plataforma Verificado18 & $1,96 \%$ & 0 & $100 \%$ \\
\hline Entidades del mundo académico & $1,3 \%$ & 0 & $100 \%$ \\
\hline Entidades del mundo civil & $1,3 \%$ & $50 \%$ & $50 \%$ \\
\hline Líderes políticos de naciones extranjeras & $1,3 \%$ & 0 & $100 \%$ \\
\hline Agencias Rusas & $1,3 \%$ & 0 & $100 \%$ \\
\hline ONG & $1,3 \%$ & 0 & $100 \%$ \\
\hline Líderes religiosos /Papa & $0,65 \%$ & 0 & $100 \%$ \\
\hline Interpol & 0,65 & 0 & $100 \%$ \\
\hline Otros & $0,65 \%$ & 0 & $100 \%$ \\
\hline
\end{tabular}

Fuente: elaboración propia con datos de verificado.mx 


\subsection{Presentación y divulgación de los resultados}

La divulgación de las comprobaciones y, en los casos necesarios, la publicación de su corrección se realiza tanto en su página Web, verificado.mx, como a través de las redes sociales, donde se distribuye bajo el sello "\#Verificado2018". Asimismo, estos materiales aparecen en el buscador Google etiquetados como: "Hecho Verificado".

En la página Web, los resultados se presentan atendiendo a la siguiente estructura: 1) inclusión de etiquetas que indican el veredicto obtenido tras la evaluación; 2) titular aclaratorio que sintetiza cuál es el contenido sometido a cuarentena; 3) primer párrafo referenciando el desmentido o corroboración de los hechos; 4) captura de pantalla con la imagen del ítem evaluado; 5) corpus con la corrección o verificación, aliñada con los datos que avalan dicha postura; 6) comentarios de los usuarios en abierto (Verificado, 2018:Web).

Asistimos, sin embargo, a la falta de coherencia y arbitrariedad de criterios ya observadas en la aplicación de las metodologías de verificación (apartado 3.1). Esta falta de uniformidad se constata en varios aspectos:

a) En la presentación de los contenidos: No todas las publicaciones que aparecen en las secciones "Noticias Falsas" o "Verificaciones" atañen a la evaluación de una información incierta: el $10 \%$ de los post corresponden, más propiamente, a materiales elaborados para ofrecer información contextual sobre una determinada polémica o comprobación, mientras que el $8 \%$ poseen una vertiente meramente educativa, brindando asesoramiento sobre, por ejemplo: "¿Qué puedes hacer y que no en la casilla electoral? (Manual de conducta para ir a votar)" o "¿Sabes qué hacer si detectas un delito el día de la campaña electoral?" Ahora bien, aun cuando estas publicaciones son de gran valor ilustrativo para el público, su inclusión en las mencionadas secciones, destinadas al análisis de declaraciones y contenidos maniqueos, indica una falta en la homogenización de criterios.

b) En el veredicto de las comprobaciones: la citada merma en la unificación de principios se observa, igualmente, en la categorización de los materiales evaluados. Dado que se entrelazan las diferentes metodologías destinadas a cada sección, la sección "Verificaciones" hace uso de las taxonomías que, en principio, sólo se aplican al apartado "Noticias Falsas".

c) En las etiquetas: Otra puntualización a tener en cuenta es la inclusión de una serie de etiquetas subsidiarias que equivalen a las categorías de los dictámenes aquí citados y que aparecen calificando las publicaciones de la Web, aun cuando dichos tags están destinados, en principio, a la distribución de contenidos en redes. Es el caso del hashtag "\#EstoSíPasó", creado 
para visibilizar en Facebook y Twitter hechos que sí tuvieron lugar, pero que también se incluye en la página de Verificado18, solapándose con el dictamen "Verdadero".

Asimismo, en un alto porcentaje de informaciones (72,27\%) no aparece citado, de manera explícita, el resultado de la comprobación. Esto ocurre cuando el veredicto de la información es "Falso": Si bien en el apartado "Verificaciones" estas aparecen marcadas con dicho tag, no así en su homólogo correspondiente a la sección "Noticias Falsas", entendemos que por equiparación entre el título de la sección y el resultado de la comprobación.

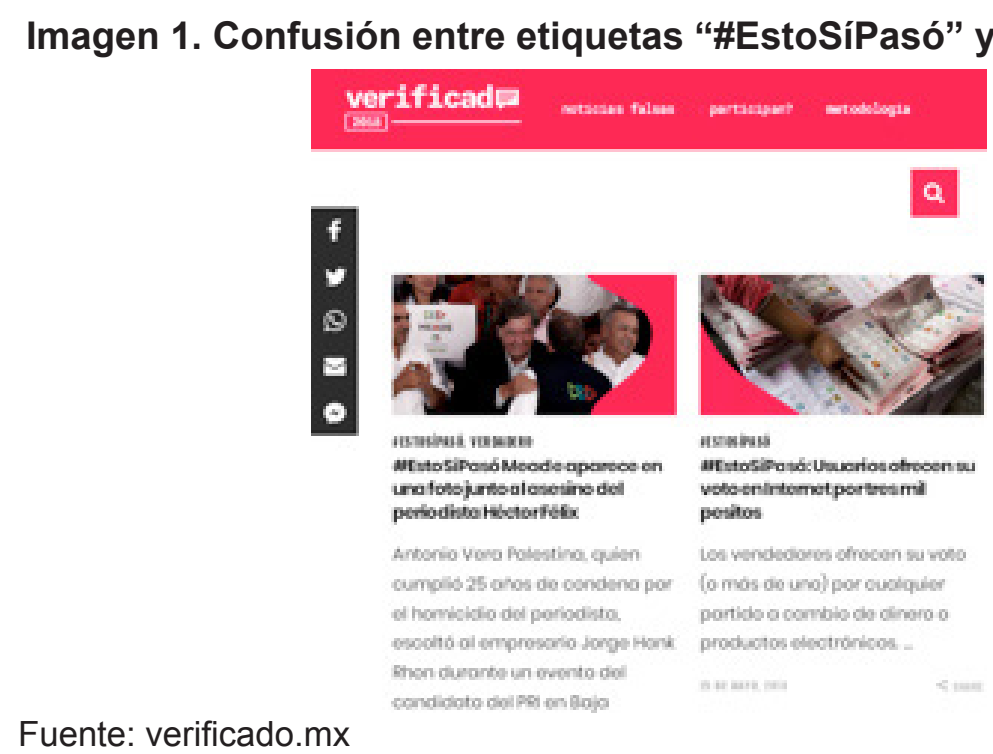

Imagen 2. Falta de homogeneización en el etiquetado: el veredicto aparece en el primer caso en el título, en el segundo como etiqueta

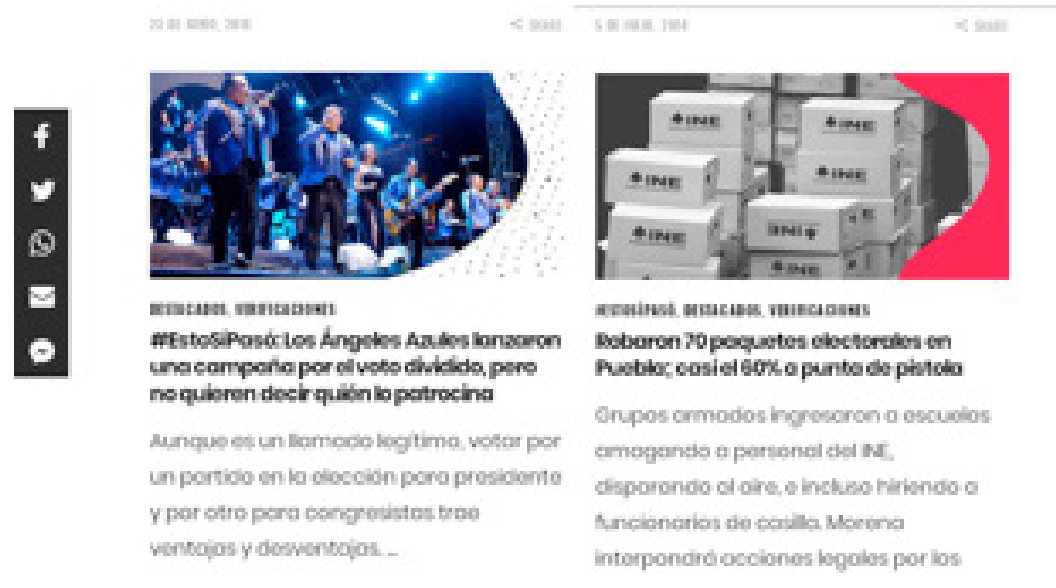

Fuente: verificado.mx 


\subsection{Multimedia, interactividad y contenidos educativos}

Verificado18 se jacta de ser "la primera iniciativa de este tipo que crea formalmente una mesa de periodismo multimedia de alta calidad" (2018:Web). No en vano, la sección "Multimedia" es la parte más mimada de su página y cuenta con contenidos de notable interés. Se trata de piezas audiovisuales, muy elaboradas y creativas que, gracias a su lenguaje sencillo y explicativo, conectan fácilmente con el público, clarificando algunos de los conceptos clave de la campaña.

La mayor parte de las entradas de este apartado (14 de 26, es decir, el 53,84\%) están destinadas a divulgar contenidos educativos y explicativos. Son piezas con un grafismo cuidado, entre las que destacan tutoriales como: "Confesiones de bots", "La Guía de 'fake news' de Verificado 2018", "Detrás de los spots", "La desinformación memética" o "Verificando memes". Incluyen, además, vídeos explicativos como: "Lo que no puedes hacer en la casilla", así como otras curiosidades como: “¿Cuánto cuesta un tuit a favor de un candidato?", "Teorías de la conspiración en las elecciones" o "EI ABC del acarreo", un interesante reportaje sobre la compra de votos en México. Entre los tutoriales con mayor impacto se encuentran: “¿Cómo verificar una foto desde tu celular?" con 1.1 millones de vistas y compartido 24.1 mil veces; y "México, elecciones y bots", con 338 mil vistas y 9.2 mil shares (2018:Web). Asimismo, se elaboran tarjetas optimizadas para el móvil en las que se explican cuestiones esenciales para comprender cómo funcionan las campañas de desinformación.

Este apartado se completa con otros diez vídeos que ayudan al usuario a contextualizar las informaciones evaluadas (38,46\% del total). Finalmente, se ofrecen dos resúmenes de los debates presidenciales, información que se suma a los comentarios a tiempo real.

En cuanto a la interactividad, la plataforma apela a la colaboración de los lectores mediante distintas etiquetas con el objetivo de que estos abandonen su role pasivo y reporten noticias dudosas. Esta interacción se produce, principalmente, a través del hashtag "\#quieroqueverifiquen" del servicio microblogging Twitter o a través de su perfil en la red social Facebook.

Además de la retroalimentación promovida por la existencia de comentarios abiertos en todas las secciones de la plataforma y de demandar la implicación de la audiencia a la hora de localizar contenidos inexactos, se busca incentivar su participación haciendo uso de otros recursos como: gifts, gráficos, transmisiones en vivo y WhatsApp. En concreto, se utiliza este sistema de mensajería instantánea en dos sentidos: a) para que los usuarios denuncien materiales dudosos y b) para establecer confirmaciones sobre contenidos falsos profusamente divulgados, mediante 
el uso de los status. Según datos de la plataforma, de todas las solicitudes de verificación que reciben semanalmente, aproximadamente 300 provienen de este canal (2018:Web).

Destaca, igualmente, la labor realizada por el equipo de redacción durante los tres debates electorales, en los que se retransmitieron las declaraciones y argumentos esgrimidos por los candidatos a través de las redes sociales, desmintiendo, a tiempo real, todas aquellas afirmaciones no constatables. Estos, a su vez, aparecían aliñados con datos que fundamentaban la corrección, toda vez que el equipo interaccionaba con aquellos usuarios que solicitaban la comprobación de algún comentario sospechoso.

\section{DISCUSIÓN Y CONCLUSIONES}

Los resultados del escrutinio esbozan una tendencia previsible: se observa una clara correlación entre las cuestiones más polémicas de la agenda electoral y los ejes temáticos sobre los cuales versan buena parte de las informaciones manipuladas. Dicha tendencia se repite cuando examinamos los actores protagonistas de las comprobaciones, hecho que está en línea como los hallazgos ya descritos en estudios precedentes, en los que se constata que aquellos agentes que ocupan "puestos de relevancia en la escala de poder son más susceptibles de ser sometidos a las propuestas de verificación" (Palau-Sampio, 2018:358). La misma prevalencia se observa claramente cuando trazamos la correspondencia entre actores implicados y el grado de veracidad de la información: en el caso de los artículos protagonizados por agentes directamente relacionados con la gestión de los comicios, tales como el INE, el promedio de veracidad de los ítems evaluados es de uno de cada dos. Mientras que, en lo que respecta a actores como los propios candidatos y partidos políticos, el porcentaje de materiales falseados es mayor: casi siete de cada diez contenidos divulgados son falsos. Así pues, el elevado número de informaciones falsas referidas tanto a informaciones como a agentes clave para el proceso electoral, avala la necesidad de un servicio de verificación de contenidos.

Ahora bien, el presupuesto de partida de esta investigación era evaluar dos aspectos básicos que condicionan, de manera directamente proporcional, la labor de las plataformas de Fact-checking: a) la transparencia en el trabajo realizado por la plataforma y b) su capacidad contextualizadora del escenario informativo. Comencemos por el primer factor, la transparencia, principio que constituye no sólo el epicentro que sustenta su razón de ser, sino que conforma un imperativo ético, suscrito por la propia Red Internacional de Fact-Checking. A este tenor, el caso estudiado se configura, en líneas generales, como un modelo capaz de rendir cuentas sobre su labor, en tanto que el proceso de autentificación de los materiales sometidos a escrutinio, esto es, la metodología seguida junto con las distintas etapas que conlleva el procedimiento, aparece 
avalada por iniciativas anteriores y todos los pasos seguidos aparecen perfectamente plasmados en su Web. Este hecho redunda, además, en su capacidad para trasladar al gran público los vericuetos de la verificación de fuentes, poniendo en valor la labor de los profesionales del periodismo. Por contra, la ya mencionada arbitrariedad en el uso de metodologías y en la posterior calificación de contenidos evaluados oscurece la labor en pro de la transparencia informativa de la plataforma. En la misma línea, se echa de menos la inclusión de datos más detallados sobre cuántas peticiones de verificaciones han recibido a través de cada medio y qué cantidad de las mismas referían a contenidos falsos. Este punto es especialmente importante a la hora de estimar la capacidad de cada canal informativo no sólo para divulgar materiales dudosos, sino para evaluar el impacto generado en el usuario.

En cuanto a su labor contextualizadora de la información, cabe destacar la sección "Multimedia", así como todas aquellas entradas que encontramos en otros apartados de la página y que poseen un alto potencial educativo. Se constata aquí el aporte de esta iniciativa a la alfabetización mediática de los ciudadanos, fortaleciendo su capacidad crítica mediante contenidos explicativos que permiten, no sólo interpretar el panorama político concreto, sino comprender las dinámicas que fomentan la desinformación. De este modo, se refuerza su conocimiento del proceso de elaboración informativa, permitiéndoles demandar una respuesta clarificadora a los medios así como interactuar, de manera informada, gracias a los recursos promovidos por la plataforma.

Empero en lo que respecta a la presentación de los resultados y, más en concreto, a los artículos publicados en su página Web, es pertinente mencionar un par de aspectos: En primer lugar, en los casos en los que nos se citan datos como el medio a través del cual se han diseminado los contenidos o cuántas veces se ha compartido una noticia, se genera una merma en la información contextual, puesto que nos impide entender la tanto la magnitud de la desinformación, ilustrada por la viralidad de estos contenidos, como los actores implicados. Prosiguiendo con la presentación de los resultados, pero moviendo el foco al esquema organizativo de su Web, el uso de las terminologías "Verificaciones" y "Noticias Falsas" en dos bloques independientes cuando, en la práctica, en ambos se evalúa el mismo género de contenido, la torna obtusa. A su vez, la utilización no homogénea de etiquetas induce a error, por lo que la página demanda una estructura más clara. Al no existir un criterio unificador y alternarse el uso de tags para una misma categoría, la búsqueda de contenidos a través de la Web se vuelve más farragosa e, incluso, puede llevar a una perdida de información, al no percatarse el usuario de que debe buscar a través de etiquetas distintas para acceder a todos los artículos clasificados bajo un mismo dictamen. 
Ahora bien, dichas puntualizaciones no deben infravalorar la labor ímproba que conlleva la verificación de contenido, más al contrario, deben traducirse como la demanda de mayores recursos adaptados a lo que una empresa de tamaña envergadura requiere, recursos que, además, puedan contribuir a su universalización.

Qué duda cabe que enseñar al público para que consuma este tipo de información de manera reflexiva y ayudar al usuario a que contextualice las informaciones eficazmente, constituyen desafíos nada desdeñables para un futuro que no han hecho sino despuntar. Retos, los aquí citados, a los que la plataforma Verificado18 responde con eficacia, convirtiéndose en un modelo susceptible de ser exportado a otros países, una vez implementandos los cambios mencionados.

\section{NOTAS}

${ }^{1}$ Afiliado a Fact-Checking Network (IFCN) https://goo.gl/8aDtHA. Su código ético se puede consultar en: https://ifcncodeofprinciples.poynter.org.

${ }^{2}$ El Sabueso cuenta con la certificación de la Red Internacional de Fact Checking del Instituto Poynter.

${ }^{3}$ Los porcentajes restantes equivalen a informaciones que no se pueden comprobar.

\section{REFERENCIAS}

Allcott, H., Gentzkow, M. (2017). Social Media and Fake News in the 2016 Election. Journal of Economic Perspectives 31 (2), 211-236.

Anderson, M., Caumont, A. (2014 October 11). How social media is reshaping news. Pew Research Center. Recuperado de: http://www.pewresearch.org/fact-tank/2014/09/24/ how-social-media-is-reshaping-news/

Así funciona \#Verificado2018 - Metodología (12 de marzo de 2018) Verificado18 Recuperado de: https://verificado.mx/metodologia/

Danzig, J. (2017 November 15). European Union or a new Soviet Union? Recuperado de: https:// goo.gl/8e $3 \times \mathrm{kw}$

European Commission (2018) A multi-dimensional approach to disinformation: Final report of the High Level Expert Group on Fake News and Online Disinformation. Luxembourg: European Union. Recuperado de:https://goo.gl/bk9NYg 
Gallant N.; Latzo-Toth, G.; Pastinelli M. (2015). Circulation de l'Information sur les Médias Sociaux pendant la Grève Étudiante de 2012 au Québec. Centre d'Étude des Médias. Recuperado de: http://www.cem.ulaval.ca/pdf/Circulationlnformation.pdf.

Graves, L. (2018). Boundaries Not Drawn: Mapping the institutional roots of the global fact-checking movement.Journalism Studies 19(5), 613-631.

Graves, L. \& Cherubini, F. (2016). The rise of fact-checking sites in Europe. Reuters Institute Digital News Report. Recuperado de: https://ora.ox.ac.uk/objects/ uuid:d55ef650-e351-4526-b942-6c9e00129ad7

Gentzkow, M., Jesse M. S.; Daniel F. S., (2016) Media Bias in the Marketplace: Theory. In Anderson, S., Waldofgel, J., \& Stromberg D., (ed.) Handbook of Media Economics, vol. 1B,

Gottfried J.; Shearer, E. (2016 April 26). News Use Across Social Media Platforms 2016. Pew Research Center. Recuperado de: goo.gl/WHEzOP.

Goel, V. (2018 May 14). In India, Facebook's WhatsApp Plays Central Role in Elections. The New York Times. Recuperado de: https://www.nytimes.com/2018/05/14/technology/whatsappindia-elections.html

Hazard Owen, L (2018). WhatsApp is a black box for fake news. Verificado 2018 is making real progress fixing that. Nieman Lab. Recuperado de: http://www.niemanlab.org/2018/06/ whatsapp-is-a-black-box-for-fake-news-verificado-2018-is-making-real-progress-fixing-that/ ICA (2017 January). Background to "Assessing Russian Activities and Intentions in Recent US Elections": The Analytic Process and Cyber Incident Attribution. Recuperado de: https:// www.dni.gov/files/documents/ICA 2017 01.pdf

Jungherr, A. (2015). Analyzing political communication with digital trace data. Switzerland: Springer. Recuperado de: https://goo.gl/8CWHZs.

Khatib, D.; Larrea Maccise, D. (12 de marzo, 2018). ¡Bienvenid@s a \#Verificado2018! Verificado18. Recuperado de https://verificado.mx/bienvenidos-a-verificado-2018/

Lotero-Echeverri, G.; Romero-Rodríguez, L. M.; Pérez-Rodríguez, M. A. (2018). Fact-checking vs. Fake news: Periodismo de confirmación como recurso de la competencia mediática contra la desinformación. index.comunicación, 8(2), 295-316.

Mathieu, D., (2015). The Continued Relevance of Reception Analysis in the Age of Social Media. Trípodos, 36, 13-34.

Najar, A. (1 julio 2018) 5 razones que hacen históricas las elecciones presidenciales en México. BBC Mundo. Recuperado de https://www.bbc.com/mundo/noticias-america-latina-43578377 
Palau-Sampio, D. (2018). Fact-checking and scrutiny of power: Supervision of public discourses in new media platforms from Latin America. Communication \& Society 31(3), 347-363.

Palau-Sampio, D. (2016). Metamorfosis de la prensa de referencia en el contexto digital: clickbait y estrategias de tabloide en Elpais.com. Comunicación y Sociedad, 29(2), 63-80. Doi: http:// doi.org/10.15581/003.29.2.sp.63-80

Panetta, K., (2017 October 3). From bots and Al to counterfeit reality and fake news, these predictions require IT leaders to pace their adoption. Gartner Top Strategic Kasey Panetta: Predictions for 2018 and Beyond. Recuperado de: https://goo.gl/cSbNp7

Restrepo, H. (2017). Clickbait: por qué está mal. Ética segura: red de ética y periodismo. Recuperado de: https://goo.gl/KhvPOy

Shin, J.; Jian, L.; Driscoll, K.; Bar, F. (2018). The diffusion of misinformation on social media: Temporal pattern, message, and source. Computers in Human Behavior, 83, 278-287. Doi: https://doi.org/10.1016/j.chb.2018.02.008

Silverman, C. (2016 November 16). This Analysis Shows how Fake Election News Stories Outperformed Real News on Facebook. Recuperado de: https://goo.gl/qv6nQO

Stencel, M.; Griffin, Riley (2018 February 22). Fact-checking triples over four years. Duke Reporters' Lab. Recuperado de: https://reporterslab.org/ the-number-of-fact-checkers-around-the-world-156-and-growing/

Subramanian, S., (2017 February 15). Inside the Macedonian Fake-News Complex. Recuperado de:https://reporterslab.org/the-number-of-fact-checkers-around-the-world-156-and-growing/ Sydell, L. (2016 November 23). We Tracked Down a Fake-News Creator in the Suburbs. Here's What We Learned. Recuperado de: https://goo.gl/hN5pZ8

Tesich, S. (1992 January 1). A Government of Lies. The Nation. Recuperado de: https://goo.gl/ dzEZqi

Townsend, T., (2016 November 22). Meet the Romanian Trump Fan behind a Major Fake News Site. Recuperado de: http://www. inc.com/tess-townsend/ending-fedtrump-facebook.html. Tufekci, Z., (2015 July 5). How Facebook's Algorithm Suppresses Content Diversity (Modestly) and How the Newsfeed Rules Your Clicks. Recuperado de: goo.gl/QI1XEd

Viner, K. (2016 July 12). How technology disrupted the truth. Recuperado de: goo.gl/glqGWM. 